\title{
Acute hyperglycemia prevents dexmedetomidine-induced preconditioning against renal ischemia-reperfusion injury ${ }^{1}$
}

\author{
Huaxin Wang', Hui Chen", Lei Wang ${ }^{\mathrm{III}}$, Lin Liu ${ }^{\mathrm{IV}}$, Min Wang ${ }^{\mathrm{IV}}$, Xiuheng Liu
}

DOI: http://dx.doi.org/10.1590/S0102-86502014001900008

${ }^{I}$ Master, Department of Urology, Renmin Hospital of Wuhan University, Wuhan, China. Main author. Conception and design of the study, acquisition and interpretation of data, manuscript writing.

IIPhD, Department of Urology, Renmin Hospital of Wuhan University, Wuhan, China. Acquisition of data, critical revision.

II'Bachelor, Department of Urology, Renmin Hospital of Wuhan University, Wuhan, China. Acquisition of data.

${ }^{\text {IV }}$ Master, Department of Urology, Renmin Hospital of Wuhan University, Wuhan, Hubei, China. Acquisition of data.

${ }^{\mathrm{v}} \mathrm{PhD}$, Full Professor, Department of Urology, Renmin Hospital of Wuhan University, Wuhan, China. Design and supervised all phases of the study, critical revision.

\section{ABSTRACT}

PURPOSE: To investigate the effects of acute hyperglycemia on dexmedetomidine-induced preconditioning against renal ischemiareperfusion injury.

METHODS: Sprague-Dawley rats were randomly arranged to the normoglycemic (NG) or hyperglycemic group (HG), with each group further divided into sham (no I/R injury), I/R (ischemia-reperfusion) and dex (given by dexmedetomidine) groups. Acute hyperglycemia was induced by intraperitoneal injection (i.p.) of $25 \%$ glucose ( $3 \mathrm{~g} / \mathrm{kg}$ ) $45 \mathrm{~min}$ before ischemia. Dexmedetomidine ( $50 \mu \mathrm{g} / \mathrm{kg}$, i.p.) was administrated $30 \mathrm{~min}$ before induction of ischemia. Renal function, histology, apoptosis, expression of Bax, Bcl-2 and phosphorylated AKT (p-AKT) were detected.

RESULTS: I/R insult significantly increased the serum levels of blood urea nitrogen and creatinine, apoptotic tubular epithelial cells, expression of Bax and $\mathrm{p}-\mathrm{AKT}$, but decreased Bcl-2 expression. All these changes were further enhanced by hyperglycemia $(\mathrm{p}<0.05)$. In hyperglycemic condition, there was no statistically difference between the $\mathrm{I} / \mathrm{R}$ group and Dex group in all the aforementioned detection indexes $(\mathrm{p}>0.05)$.

CONCLUSION: Acute hyperglycemia attenuates dexmedetomidine-induced preconditioning against renal ischemia-reperfusion injury in non-diabetic rats.

Key words: Hyperglycemia. Ischemia. Reperfusion. Kidney. Dexmedetomidine. Rats. 


\section{Introduction}

Ischemia-reperfusion ( $\mathrm{I} / \mathrm{R}$ )-induced renal injury remains a leading cause of delayed graft dysfunction and chronic allograft nephropathy after kidney transplantation ${ }^{1}$. Thus, approaches to lessen I/R injury have been extensively studied, and administration of pharmacologic agents such as anesthetics have been shown to be protective against renal $\mathrm{I} / \mathrm{R}$ injury ${ }^{2,3}$. Dexmedetomidine, a commonly used anesthetic, could exert renoprotective effects on normoglycemic animals when administered before renal $\mathrm{I} / \mathrm{R}^{4-6}$.

Hyperglycemia is an independent factor contributing to the adverse outcomes of renal transplantation ${ }^{7}$. Transient hyperglycemia, not uncommon in non-diabetic patients after renal transplantation, could accentuate renal ischemic injury, apoptosis, antigen presentation and inflammatory responses which might increase the risk of graft rejection ${ }^{8}$. Previous studies have proved that hyperglycemia could render the cardiopreotective effects of anesthetic preconditioning counterproductive ${ }^{9,10}$. Besides, two anesthetics, i.e. propofol and isoflurane, lost their protective effect of renal $I / R$ injury during transient hyperglycemia ${ }^{11}$. Nevertheless, it is still unclear whether acute hyperglycemia before I/R could compromise the protective effects of dexmedetomidine-induced preconditioning. The major purpose of this study was to investigate whether the impact of acute hyperglycemia were refractory to protection induced by dexmedetomidine preconditioning in a rat model.

\section{Methods}

This project was approved by the committee of experimental animals, and the procedures were carried out according to the routine animal-care guidelines. All experimental procedures complied with the Guide for the Care and Use of Laboratory Animals.

Eighty-four adult male Sprague-Dawley rats (weighing 250-300g) were from the Center of Experimental Animal in Medical College, Wuhan University. All animals were anesthetized by $45 \mathrm{mg} / \mathrm{kg}$ pentobarbital sodium body weight, i.p. Renal warm ischemia was induced by placing a nontraumatic microvascular clamp on the left renal artery and vein for $45 \mathrm{~min}$ after right nephrectomy, followed by $24 \mathrm{~h}$ reperfusion. Successful ischemia or reperfusion was judged by observing the change in tissue color from red to dark blue or from dark blue to bright red respectively. Blood samples were obtained from the tail vein or the inferior vena cava.

\section{Groups and drug administration}

Eighty-four rats were divided into two different treatment schedules: Schedule A was designed to evaluate biochemical parameters, histopathological and apoptotic proteins; Schedule B was designed to evaluate the levels of total AKT and phosphorylated AKT (p-AKT) levels.

In schedule $\mathrm{A}$, animals were randomly assigned to six groups $(\mathrm{n}=8$, Table 1$)$ with three groups of hyperglycemia $(\mathrm{HG})$ receiving $25 \%$ glucose $(3 \mathrm{~g} / \mathrm{kg}$, i.p.), whilst the other three groups were normoglycemia $(\mathrm{NG})$ given the same amount of saline 45 min prior to ischemia.

TABLE 1 - Experimental groups.

\begin{tabular}{ccccc}
\hline Group & Saline & Glucose & I/R & Dex \\
\hline NG-Sham $(n=8)$ & + & - & - & - \\
NG-I/R $(n=8)$ & + & - & + & - \\
NG-Dex $(n=8)$ & + & - & + & + \\
HG-Sham $(n=8)$ & - & + & - & - \\
HG-I/R $(n=8)$ & - & + & + & - \\
HG-Dex $(n=8)$ & - & + & + & + \\
\hline
\end{tabular}

NG: normoglycemia, HG: hyperglycemia, I/R: Ischemia-reperfusion, Dex: dexmedetomidine.

Sham groups with or without hyperglycemia underwent the identical surgical procedures, with the exception of renal clamping. I/R groups with normoglycmia or hyperglycemia underwent renal ischemia for $45 \mathrm{~min}$ followed by reperfusion. In either NG-Dex or HG-Dex group, dexmedetomidine $(50 \mu \mathrm{g} / \mathrm{kg}$, i.p.) was administrated $30 \mathrm{~min}$ before I/R.

In schedule $\mathrm{B}$, animals were also randomly divede into six groups $(n=6)$ as schedule A. Left kidneys were harvested at 15 min after starting reperfusion.

\section{Biochemical parameters}

Blood glucose concentrations were monitored from the tail vein blood before glucose or saline intraperitoneal administration (T0), before ischemia (T1), and 24h after reperfusion (T2).

Renal function was assessed by serum creatinine $(\mathrm{Cr})$ and plasma urea concentrations after $24 \mathrm{~h}$ reperfusion. Blood samples collected via the inferior vena cava were centrifuged $(4.000 \times \mathrm{g}$ for $10 \mathrm{~min}$ ) to separate the serum and frozen until $\mathrm{Cr}$ and urea concentrations were measured. 


\section{Histopathological evaluation and preparation of protein}

At $24 \mathrm{~h}$ after reperfusion, the left kidney of schedule A was excised and sectioned longitudinally into two fragments. One fragment was fixed in buffered formalin and embedded in paraffin, stained with hematoxylin-eosin, and examined under a light microscope by a pathologist unaware of the grouping. A grading scale of $0-4$, as outlined by Jablonski et al. ${ }^{12}$ was used for the histopathologic assessment of $\mathrm{I} / \mathrm{R}$ induced damage of the proximal tubules. In addition, Tunel assay was performed to detect apoptosis in situ cell death according to the manufacturer's instructions (TUNEL kit). The results of staining were analyzed and evaluated with American Image-Pro Plus software. The percentage of positive cells with TUNEL staining in five $400 \times$ sights served as apotosis index (AI).

The other fragment and the left kidney of schedule B were stored at $-80 \square$ until pulverized and dissolved in protein lysis buffer. The solution was centrifuged at $12.000 \mathrm{~g}$ for $5 \mathrm{~min}$ at $4 \square$, and the supernatant was aliguoted, snap frozen, and stored at $-80 \square$ until use to analyze. Bax, Bcl-2, total levels of Akt and p-AKT were analysed as described by our previous study ${ }^{13}$ by sodium dodecyl sulfate-polyacrylamide gel electrophoresis
(SDS-PAGE) using antibodies obtained from Cell Signaling Inc (Boston, MA, USA). Glyceraldehyde-3-phosphatedehydrogenase (GAPDH) was used as the internal control. Protein concentrations were determined by densitometry values and normalized to GAPDH.

\section{Statistical analysis}

Data were expressed as mean \pm standard deviation. Statistical analysis was performed using analysis of variance followed by the Dunn post hoc test. Values of $p<0.05$ were recognized as statistically significant.

\section{Results}

\section{Plasma glucose levels}

Baseline plasma glucose (T0) did not differ among 6 groups. While the increase of plasma glucose concentrations was significant in 3 HG-groups after glucose injection (T1). However, serum glucose concentrations returned to normal at the $24 \mathrm{~h}$ after reperfusion (Table 2).

TABLE 2 - The plasma glucose levels of rats.

\begin{tabular}{ccccccc}
\hline & NG-Sham & NG-I/R & NG-Dex & HG-Sham & HG-I/R & HG-Dex \\
\hline T0 & $5.79 \pm 0.61$ & $5.83 \pm 0.58$ & $5.74 \pm 0.70$ & $5.71 \pm 0.73$ & $5.80 \pm 0.46$ & $5.77 \pm 0.66$ \\
T1 & $7.05 \pm 0.700$ & $7.19 \pm 0.75$ & $7.04 \pm 0.63$ & $19.21 \pm 3.00^{* \#}$ & $19.43 \pm 2.14^{* \#}$ & $18.94 \pm 2.34^{* \#}$ \\
T2 & $5.76 \pm 0.66$ & $5.90 \pm 0.44$ & $6.01 \pm 0.24$ & $5.86 \pm 0.57$ & $6.11 \pm 0.50$ & $6.04 \pm 0.59$ \\
\hline
\end{tabular}

${ }^{*} \mathrm{p}<0.01$ vs. corresponding T0; ${ }^{*} \mathrm{p}<0.01$ vs. corresponding $\mathrm{T} 1$ of the NG groups, respectively. T0: baseline, T1: before ischemia; T2: $24 \mathrm{~h}$ after reperfusion.

\section{Renal function and renal histology}

Rats subjected to I/R injury showed significant increases in urea and $\mathrm{Cr}$ compared with sham rats. Moreover, the urea and Cr were higher in HG-I/R group than in NG-I/R group. The renal function changes induced by $\mathrm{I} / \mathrm{R}$ were significantly improved by dexmedetomidine treatment in NG-Dex group compared with NGI/R group. However, there was no significant difference in renal function between HG-Dex group and HG-I/R group (Figure 1).
Animals subjected to $\mathrm{I} / \mathrm{R}$ injury showed significant more severe tubular damage (including widespread degeneration of tubular dilation, tubular cell swelling, pyknotic nuclei, severe tubular necrosis) compared to sham rats. Compared with NG-I/R group, only mild damage in renal histological architecture was seen in the NG-Dex group. Nevertheless, there was no obvious difference between HG-I/R group and HG-Dex group (Figure 2). 

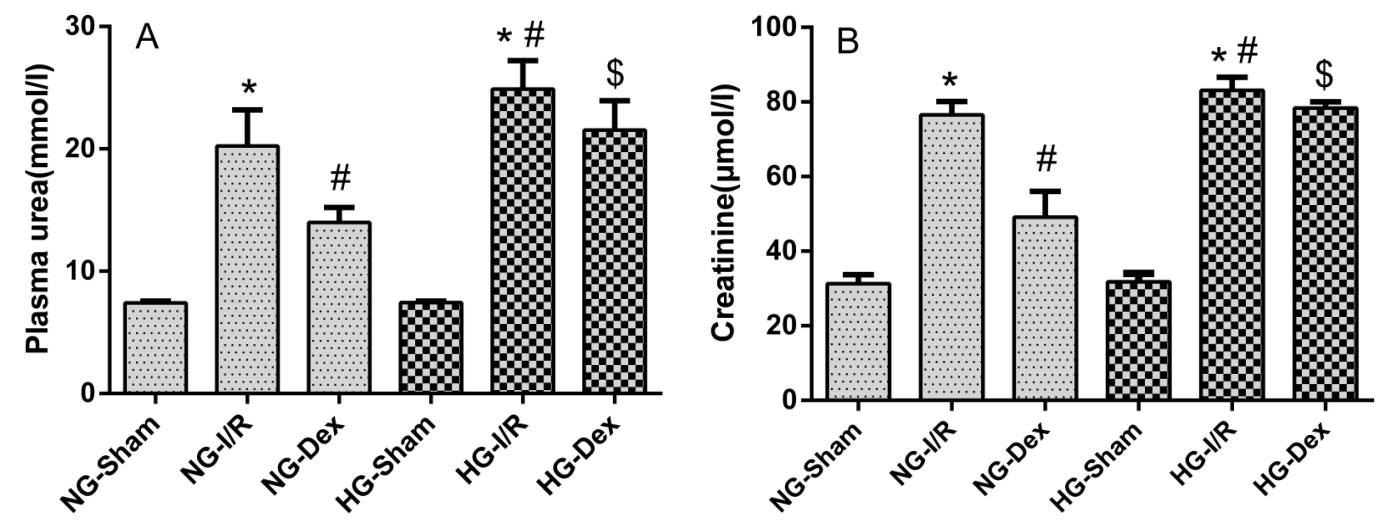

FIGURE 1 - Effects of dexmedetomidine on alterations of renal fuction following renal I/R injury. Plasma urea (A) and serum creatinine (B) were measured $24 \mathrm{~h}$ after renal ischemia-reperfusion injury. ${ }^{*} \mathrm{p}<0.05 \mathrm{vs}$. NG-Sham group or HG-Sham group; ${ }^{\#} \mathrm{p}<0.05 \mathrm{vs}$. NG-I/R group; ${ }^{\$} \mathrm{p}<0.05$ vs. NG-Dex group.
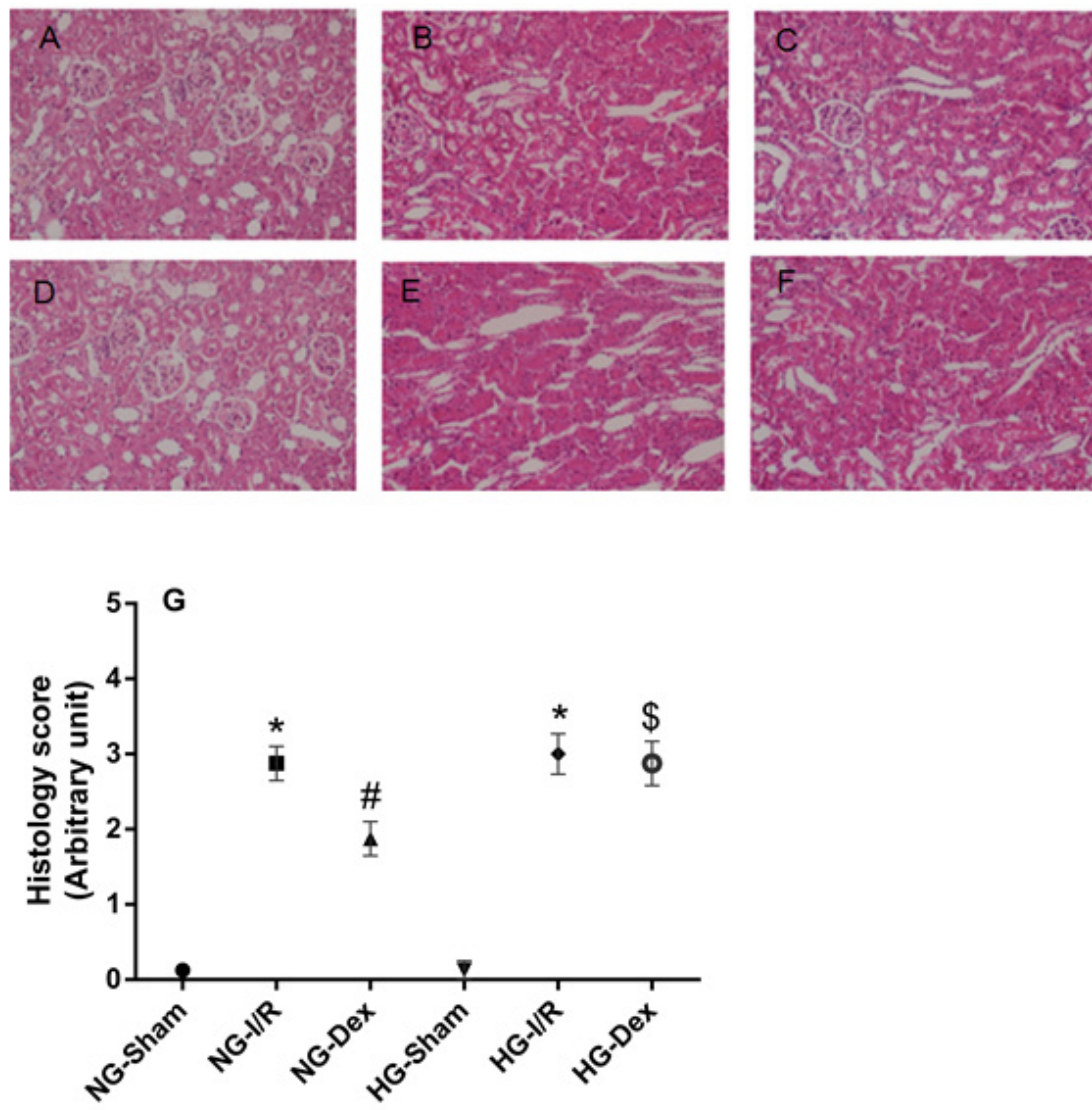

FIGURE 2 - The sham groups did not show any morphological changes (A,D). The kidneys of untreated ischemia rats, including HGDex group showed tubular cell swelling, cellular vacuolization, and moderate to severe necrosis (B,E,F). The NG-Dex group shows slight edema of the tubular cells $(\mathbf{C})$. All hematoxylin and eosin $\times 200$. Quantification of histological scoring $(\mathbf{G}) . * \mathrm{p}<0.05 \mathrm{vs}$. NG- Sham group or HG-Sham group; ${ }^{\#} \mathrm{p}<0.05$ vs. NG-I/R group; ${ }^{\mathrm{p}} \mathrm{p}<0.05$ vs. NG-Dex group. 


\section{Apoptosis and apoptosis-related proteins}

TUNEL staining assay can stain the positive apoptotic cells brown. TUNEL assay showed an increase in the number of positive cells after I/R compared with sham rats. Moreover, the number of positive cells were more in HG-I/R group than in NG$\mathrm{I} / \mathrm{R}$ group. The changes induced by $\mathrm{I} / \mathrm{R}$ were significantly less by dexmedetomidine treatment in NG-Dex group compared with NG-I/R group. However, there was no obvious difference between HG-Dex group and HG-I/R group (Figure 3).
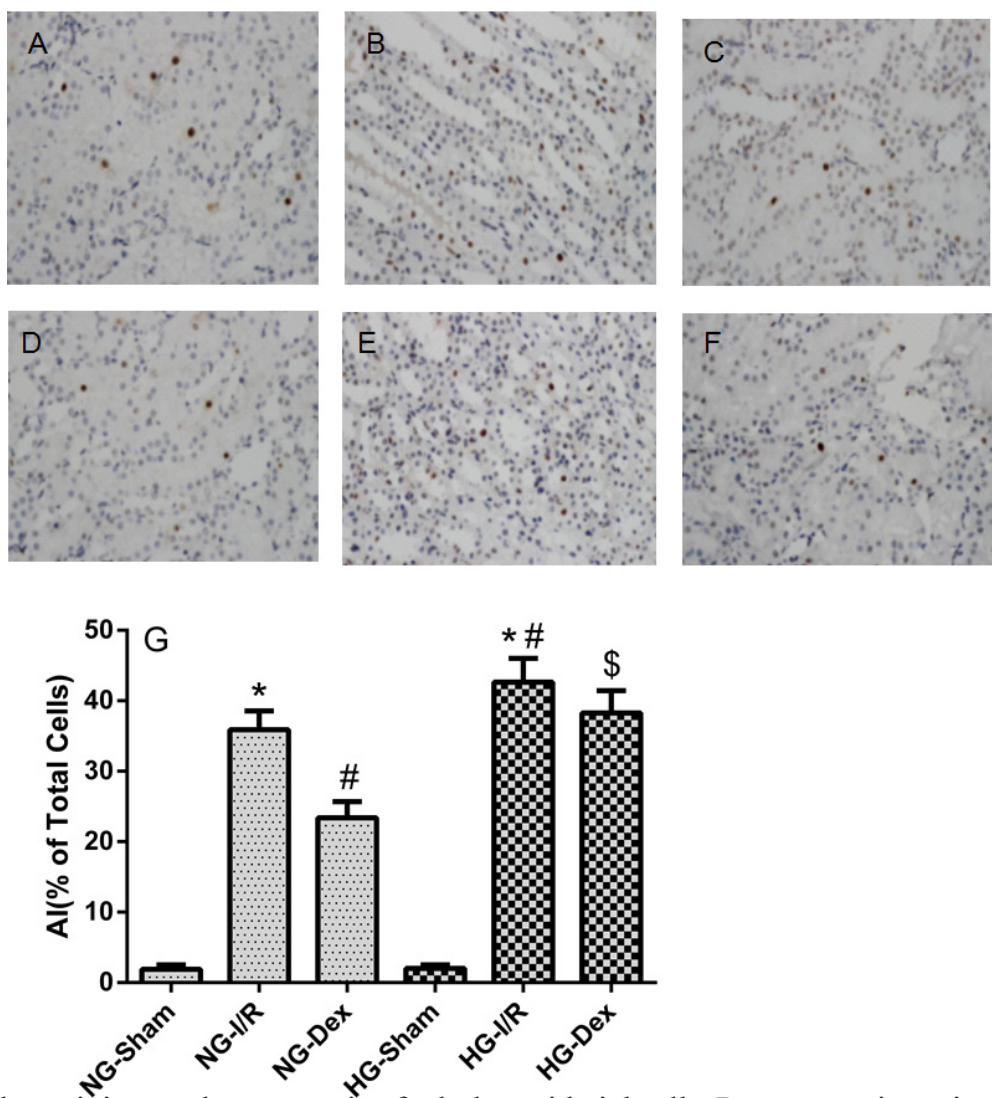

FIGURE 3 - Effects of dexmedetominine on the apoptosis of tubular epitheial cells. Representative microphotographs were taken from the kidneys of the NG-Sham (A), NG-I/R (B), NG-Dex (C), HG-Sham (D), HG-I/R (E), HG-Dex (F) groups at the time point of 24h after renal $\mathrm{I} / \mathrm{R}$ in rats. Apoptosis was evaluated by TUNEL staining. Quantification of TUNEL positive cells was counted (G). AI: apotosis index. Bars represent means $\pm \mathrm{SE}$; ${ }^{*} \mathrm{p}<0.05$ vs. NG- Sham group or HG-Sham group; ${ }^{*} \mathrm{p}<0.05$ vs. NG-I/R group; ${ }^{\$} \mathrm{p}<0.05$ vs. NG-Dex group.

Ischemia-reperfusion increased expression of the decreased and expression of Bcl-2 increased significantly proapoptotic proteins, Bax and decreased expression of the compared to the NG-I/R group. In the HG-Dex group, Bax antiapoptotic protein, Bcl-2, in both the NG-I/R group and level increased and Bcl-2 level decreased compared to the NGthe HG-I/R group. In the NG-Dex group, expression of Bax Dex group (Figure 4).
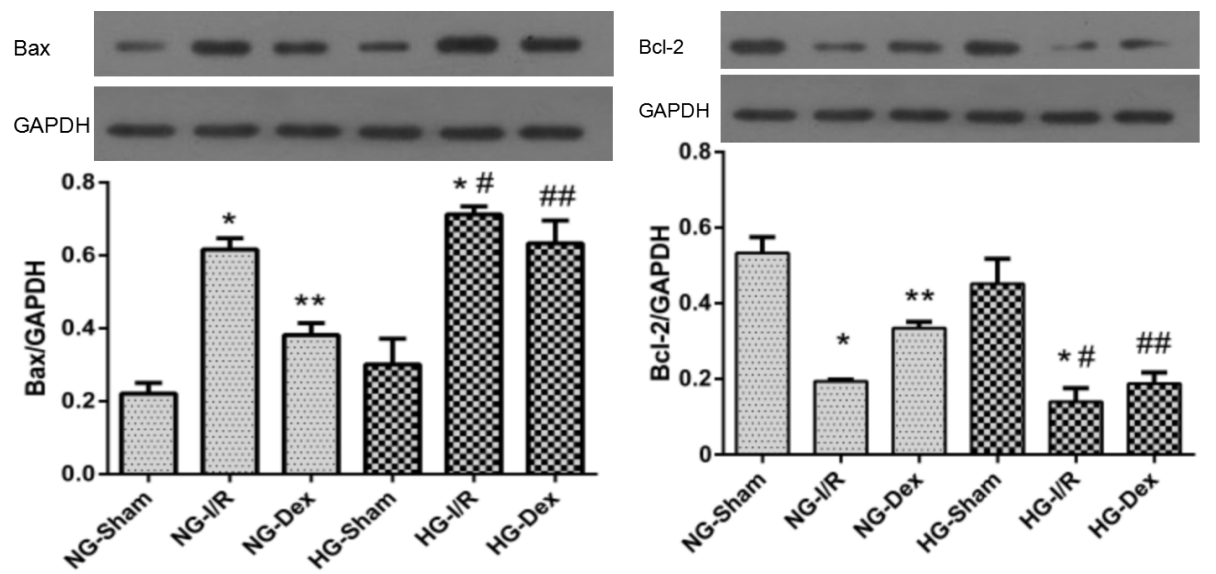

FIGURE 4 - Western blot analysis of Bax and Bcl-2 expression. " $\mathrm{p}<0.05$ vs. NG-Sham group or HG-Sham group; ${ }^{* *} \mathrm{p}<0.05$ vs. NG-I/R group; ${ }^{\#} \mathrm{p}<0.05$ vs. NG-I/R group; ${ }^{\#} \mathrm{p}<0.05$ vs. NG-Dex group. 
Phosphorylated AKT was similar between NG-Sham group and HG-Sham group. Ischemia-reperfusion significantly increased the levels of p-AKT in both NG-I/R group and HG$\mathrm{I} / \mathrm{R}$ group. And the expression of p-AKT were higher in $\mathrm{HG}-\mathrm{I} / \mathrm{R}$ group than in NG-I/R group. In the NG-Dex group, the levels of p-AKT were significant increase than in the $\mathrm{NG-I/R}$ group. However, there was no difference between the HG-Dex and HGI/R group (Figure 5).

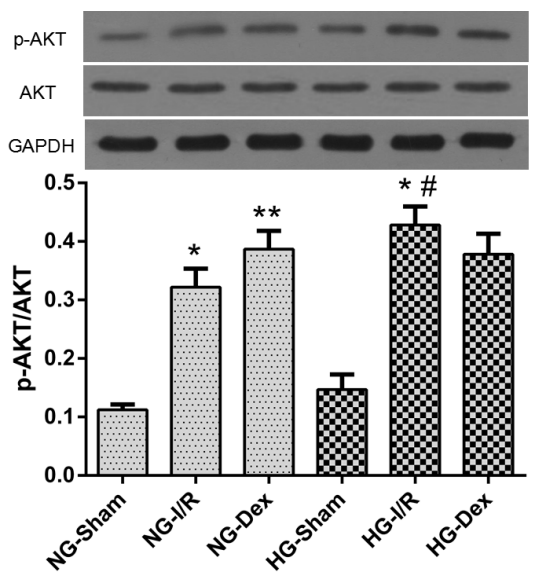

FIGURE 5 - Western blot analysis of p-AKT expression. ${ }^{*} \mathrm{p}<0.05$ vs. NG- Sham group or HG-Sham group; ${ }^{* *} \mathrm{p}<0.05$ vs. NG-I/R group; ${ }^{\mathrm{p}}<0.05$ vs. NG-I/R group.

\section{Discussion}

Although dexmedetomidine is powerful against I/R injury intervention and appears safe, its feasibility need to be discussed. Our study showed that acute hyperglycemia abolished the renal protective effects of dexmedetomidine. Moreover, we demonstrated for the first time that the protective effects of dexmedetomidine treatment was lost in acute hyperglycemic rats after renal I/R injury.

Dexmedetomidine has been described as an efficacious and safe adjunct in many clinical applications. Recent studies have showed that dexmedetomidine could reduce the renal I/R injury, by inhibiting Janus kinase/signal transducer and activator of transcription (JAK/STAT) pathway ${ }^{4}$ or activating cell survival signal p-AKT ${ }^{5}$. Koca et al. ${ }^{14}$ showed that dexmedetomidine also attenuated sepsis-induced kidney injury and apoptosis in the rat model of sepsis. Acute perioperative hyperglycemia is known as an established risk factor for many pathologic processes. Previous report showed that there were more severe reperfusion-induced injuries in the kidneys of perioperative hyperglycemic rats ${ }^{15,16}$, but the reason remains unclear. This study supported and extended these previous findings, demonstrating that hyperglycemia could accentuate renal ischemic injury and dexmedetomidine could reduce the renal $\mathrm{I} / \mathrm{R}$ injury. However, treatment with dexmedetomidine was invalided in the acute hyperglycemic I/R rat models. In our study, renal I/R injury induced more severe renal dysfunction, and morphology changes in acute hyperglycemic rats. Meanwhile, the protective effect of dexmedetomidine was lost in hyperglycemic rats, which was proved by renal function and morphology changes.

Renal apoptosis is an important factor in the development of acute renal failure after I/R injury. The Bax/Bcl-2 family of proteins highlights the complexity of cellular biology. Bax is a proapoptotic protein with a role of promoting cytochrome release, while Bcl-2 conversely, is an antiapoptotic protein which can prevent cytochrome c release from mitochondria and protect DNA from fragmentation. AKT, a serine/threonine specific protein kinase, is known regulators of cell survival, and it plays a central role in the signaling pathways regulating metabolism and cellular transformation. It has been shown that AKT signaling was critical to recovery from renal I/R injury ${ }^{17}$. A recent work demonstrated that dexmedetomidine increased the expression of $\mathrm{p}$-AKT in a human kidney cell line ${ }^{5}$. In order to further clarify the reason of lost, we investigated the expressions of key apoptotic-related molecules. This study supported previous findings and further supported our opinion. Our study showed that dexmedetomidine increased the levels of anti-apoptotic Bcl-2 protein and inhibited the expression of Bax protein. But these effects were weak in hyperglycemic rats. Therefore, we speculated that in hyperglycemic rats, the severe apoptosis might be refractory to anti-apoptotic effect by dexmedetomidine. In addition, dexmedetomidine treatment also increased expression of $\mathrm{p}$-AKT in normoglycemic rats, whereas transient hyperglycemia inhibited the expression of $\mathrm{p}-\mathrm{AKT}$ in hyperglycemic rats after treatment with dexmedetomidine. Therefore, we speculated that hyperglycemia might prevent protective effects of dexmedetomidine by inhibiting phosphorylation of AKT.

Several aspects of the present study deserve further discussion. We used only one dose to investigate the effect of acute hyperglycemia. The dose $(50 \mu \mathrm{g} / \mathrm{kg}$, i.p.) referred to previous literature in rats ${ }^{4,14}$. Whether the larger dose of dexmedetomidine decreases renal I/R injury under hyperglycemia may need to be elucidated in further research. In addition, it is possible that dexmedetomidine treatment might only leads to long-term protection in hyperglycemic rats. Thus, the long-term consequences of dexmedetomidine may need to be investigated in further study. 


\section{Conclusion}

Acute transient hyperglycemia prevents dexmedetomidine-induced preconditioning against renal ischemiareperfusion injury in non-diabetic rats.

\section{References}

1. Snoeijs MG, van Heurn LW, Buurman WA. Biological modulation of renal ischemia-reperfusion injury. Curr Opin Organ Transplant. 2010 Apr;15(2):190-9. doi: 10.1097/MOT.0b013e32833593eb.

2. Dogan Z, Yuzbasioglu MF, Kurutas EB, Yildiz H, Coskuner I, Senoglu N, Oksuz H, Bulbuloglu E. Thiopental improves renal ischemia-reperfusion injury. Ren Fail. 2010 Jan;32(3):391-5. doi: 10.3109/08860221003611752.

3. Mutoh J, Ohsawa M, Hisa H. Effect of naloxone on ischemic acute kidney injury in the mouse. Neuropharmacology. 2013 Aug;71:108. doi: 10.1016/j.neuropharm.2013.03.001.

4. Si Y, Bao H, Han L, Shi H, Zhang Y, Xu L, Liu C, Wang J, Yang X, Vohra A, Ma D. Dexmedetomidine protects against renal ischemia and reperfusion injury by inhibiting the JAK/STAT signaling activation. J Transl Med. 2013 Jun 9;11(1):141. PPMID: 23759023.

5. Gu J, Sun P, Zhao H, Watts HR, Sanders RD, Terrando N, Xia P, Maze M, Ma D. Dexmedetomidine provides renoprotection against ischemia-reperfusion injury in mice. Crit Care. 2011 Jun 24;15(3):R153. doi: 10.1186/cc10283.

6. Gonullu E, Ozkardesler S, Kume T, Duru LS, Akan M, Guneli ME, Ergur BU, Meseri R, Dora O. Comparison of the effects of dexmedetomidine administered at two different times on renal ischemia/reperfusion injury in rats. Braz J Anesthesiol. 2014 MayJun;64(3):152-8. doi: 10.1016/j.bjane.2013.06.002.

7. Parekh J, Roll GR, Feng S, Niemann CU, Hirose R. Peri-operative hyperglycemia is associated with delayed graft function in deceased donor renal transplantation. Clin Transplant. 2013 JulAug;27(4):E424-30. doi: 10.1111/ctr.12174.

8. Hekmat R, Eshraghi H. Correlation of pretransplant hyperglycemia and delayed graft function in kidney transplantation. Iran J Kidney Dis. 2010 Apr;4(2):147-52. PMID: 20404427.

9. Kim HS, Kim SY, Kwak YL, Hwang KC, Shim YH. Hyperglycemia attenuates myocardial preconditioning of remifentanil. J Surg Res. 2012 May 15;174(2):231-7. doi: 10.1016/j.jss.2011.01.018.

10. Canfield SG, Sepac A, Sedlic F, Muravyeva MY, Bai X, Bosnjak ZJ. Marked hyperglycemia attenuates anesthetic preconditioning in human-induced pluripotent stem cell-derived cardiomyocytes. 2012 Oct;117(4):735-44. PubMed PMID: 22820846.

11. Carraretto AR, Vianna FP, Castiglia YM, Golim MA, Souza AV, Carvalho LR, Deffune E, Vianna PT. Do propofol and isoflurane protect the kidney against ischemia/reperfusion injury during transient hyperglycemia? Acta Cir Bras. 2013 Mar;28(3):161-6. PubMed PMID: 23503855.

12. Jablonski P, Howden BO, Rae DA, Birrell CS,. Marshall VC, Tange $\mathrm{J}$, An experimental model for assessment of renal recovery from warm ischemia. Transplantation. 1983 Mar;35(3):198-204. PMID: 6340272.
13. Chen H, Xing B, Liu X, Zhan B, Zhou J, Zhu H, Chen Z. Ischemic postconditioning inhibits apoptosis after renal ischemia/reperfusion injury in rat. Transpl Int. 2008 Apr;21(4):364-71. PMID: 18069925.

14. Koca U, Olguner CG, Ergur BU, Altekin E, Tasdogen A, Duru S, Girgin P, Gunduz K, Cilaker MS, Guzeldag S, Akkus M. The effects of dexmedetomidine on secondary acute lung and kidney injuries in the rat model of intra-abdominal sepsis. Scientific World J. 2013;2013:292687. doi: 10.1155/2013/292687.

15. Podrazik RM, Natale JE, Zelenock GB, D’Alecy LG. Hyperglycemia exacerbates and insulin fails to protect in acute renal ischemia in the rat. J Surg Res. 1989 Jun;46(6):572-8. PMID: 2659896.

16. Hirose $\mathrm{R}, \mathrm{Xu} F$, Dang $\mathrm{K}$, Liu T, Behrends M, Brakeman PR, Wiener-Kronish J, Niemann CU. Transient hyperglycemia affects the extent of ischemia-reperfusion-induced renal injury in rats. Anesthesiology. 2008 Mar;108(3):402-14. doi: 10.1097/ ALN.0b013e318164cff8.

17. Satake A, Takaoka M, Nishikawa M, Yuba M, Shibata Y, Okumura K, Kitano K, Tsutsui H, Fujii K, Kobuchi S, Ohkita M, Matsumura Y. Protective effect of 17 beta-estradiol on ischemic acute renal failure through the PI3K/Akt/eNOS pathway. Kidney Int. 2008 Feb;73(3):308-17. PMID: 18004295.

\section{Correspondence:}

Xiuheng Liu

Department of Urology, Renmin Hospital of Wuhan University Jiefang Road 238

Wuhan, Hubei, China, 430060

Phone: (86)027-88041911(82033)

1xiuheng@163.com

Received: Aug 19, 2014

Review: Oct 17, 2014

Accepted: Nov 21, 2014

Conflict of interest: none

Financial source: none

${ }^{1}$ Research performed at Key Laboratory, Hubei Province for Digestive System Disease, Department of Gastroenterology, Renmin Hospital, Wuhan University, Hubei, China. 